\title{
Three dimensional viscoelastic medium under thermal shock
}

\begin{abstract}
Abhik Sur* and M. Kanoria
Department of Applied Mathematics, University of Calcutta, 92 A.P.C. Road, Kolkata-700009, West Bengal, India

\begin{tabular}{l}
\hline A R T I C L EI N F O \\
\hline Article history: \\
Received 6 March, 2016 \\
Accepted 23 June 2016 \\
Available online \\
23 June 2016 \\
\hline Keywords: \\
Generalized thermoelasticity \\
Dual-phase-lag thermoelastic \\
model \\
Kelvin-Voigt-model \\
Finite wave speed \\
Normal mode analysis \\
\end{tabular}

\section{A B S T R A C T}

This article deals with the thermoelastic interaction in a three-dimensional homogeneous and isotropic viscoelastic medium under the Dual-phase-lag model of generalized thermoelasticity. The resulting non-dimensional coupled equations are applied to a specific problem of a halfspace whose surface is traction-free and is subjected to a time-dependent thermal shock. The analytical expressions for the displacement components, stress, temperature and strain are obtained in the physical domain by employing normal mode analysis. These expressions are also calculated for a copper-like material and have been depicted graphically. Discussions have been made to highlight the effect of viscosity on the studied field. The phenomenon of a finite speed of propagation is observed for each field. Also, if the effect of viscosity is neglected, the results agree with the existing literature.
\end{abstract}

(C) 2016 Growing Science Ltd. All rights reserved.

\section{Introduction}

Linear viscoelasticity has been an important area of research since the period of Maxwell, Boltzman, Voigt and Kelvin. Valuable information regarding linear viscoelasticity theory may be obtained in the books of Gross (1953), Alfery and Gurnee (1956), Ferry (1970), Bland (1960) and Lakes (1998). Many researchers like Biot (1954, 1955), Gurtin and Sternberg (1962), Iiioushin and Pobedria (1970), Tanner (1988) have contributed notably on thermoviscoelasticity. Freudenthal (1954) have pointed out that most of the solids, when subjected to dynamic loading, exhibit viscous effects.

The Kelvin-Voigt model is one of the macroscopic mechanical model often used to describe the viscoelastic behavior of a material. The model represents the delayed elastic response subjected to stress when the deformation is time dependent but recoverable (Sur and kanoria, 2014a; Sur and Kanoria, 2014b). The dynamic interaction of thermal and mechanical fields in solids has great practical applications in modern aeronautics, astronautics, nuclear reactors and high-energy accelerators, for

* Corresponding author.

E-mail addresses: abhiksur4@gmail.com (A. Sur) 
example. Biot (1956) formulated the coupled thermoelasticity theory to eliminate the paradox inherent in the classical uncoupled theory that elastic deformation has no effect on the temperature. The field equations for both the theories are of a mixed parabolic-hyperbolic type, which predict infinite speeds for thermoelastic signals, contrary to physical observations. Hetnarski and Ignaczak (1999) examined five generalizations to the coupled theory of thermoelasticity. The first generalization is due to Lord and Shulman (1967) who formulated the generalized thermoelasticity theory involving one relaxation time. This theory is referred to as L-S theory or extended thermoelasticity theory (ETE) in which the Maxwell-Cattaneo law replaces the Fourier law of heat conduction by introducing a single parameter that acts as a relaxation time. The second generalization to the coupled theory of thermoelasticity is due to Green and Lindsay (1972), called G-L theory or the temperature-rate dependent theory (TRDTE), which involves two relaxation times. Problems concerning these generalized theories such as ETE and TRDTE have been studied by Chandrasekharaiah (1986), Ignaczak (1989). Muller (1971) proposed an entropy production inequality that led to restrictions on a class of constitutive equations. A generalization of this inequality was developed by Green and Laws (1972). Green and Lindsay obtained a modified version of the constitutive equations. These equations were independently obtained by Suhubi (1975). For a review, works of Ignaczak (1989) may be mentioned where presentation of the two theories and some important results are achieved in this field.

The third generalization to the coupled theory of thermoelasticity is known as low-temperature thermoelasticity introduced by Hetnarski and Ignaczak (1996), called the H-I theory. This model is characterized by a system of non-linear field equations. Low-temperature non-linear models of heat conduction that predict wave-like thermal signals and which are supposed to hold at low temperatures have also been proposed and studied in some works by Kosinski (1989) and Kosinski and Cimmmelli (1997).

The fourth generalization to the coupled theory is concerned with the thermoelasticity theory without energy dissipation (TEWOED) introduced by Green and Naghdi (1991, 1993), referred to as G-N theory of type II in which the classical Fourier law is replaced by a heat flux rate-temperature gradient relation. The heat transport equation does not involve a temperature-rate term and as such this model admits undamped thermoelastic waves in thermoelastic material. In the context of linearized version of this theory, theorem on uniqueness of solutions has been established by Chandrasekharaiah (1996a, 1996b). The fourth generalization of the thermoelasticity theory developed by Green and Naghdi also involves a heat conduction law, which includes the conventional law and one that involves the thermal displacement gradient among the constitutive variables. This model is referred to as the GN model III which involves dissipation of energy in general and admits damped thermoelastic waves. Taheri et al. (2005) have employed Green-Naghdi theories of type II and type III to study the thermal and mechanical waves in an annulus domain. Mallik and Kanoria (2007) have studied one dimensional thermoelastic disturbance in an isotropic functionally graded medium in the context of generalized thermoelasticity without energy dissipation. Problems concerning these theory have been studied by many authors such as Bandopadhyay and Roychoudhuri (2005), Roychoudhuri and Bandopadhyay (2005), Kar and Kanoria (2009b), Banik et al. (2007, 2009), Roychoudhuri and Dutta (2005), Mallik and Kanoria $(2008,2009)$.

The fifth generalization to the thermoelasticity theory is known as the dual phase lag model developed by Tzou (1995) and Chandrasekharaiah (1998). Tzou (1995) considered micro-structural effects into the delayed response in time in the macroscopic formulation by taking into account that the increase of the lattice temperature is delayed due to phonon-electron interactions on the macroscopic level. A macroscopic lagging (or delayed) response between the temperature gradient and the heat flux vector seems to be a possible outcome due to such progressive interactions. Tzou (1995) introduced two-phase lags to both the heat flux vector and the temperature gradient and considered a constitutive equation to describe the lagging behavior in the heat conduction in solids. According to this model, 
classical Fourier's law $\vec{q}=-k \vec{\nabla} T$ has been replaced by $\vec{q}\left(P, t+\tau_{q}\right)=-K \vec{\nabla} T\left(P, t+\tau_{T}\right)$, where the temperature gradient $\vec{\nabla} T$ at a point $P$ of the material at time $t+\tau_{T}$ corresponds to the heat flux vector $\vec{q}$ at the same point at time $t+\tau_{q}$. Here $K$ is the thermal conductivity of the material. The delay time $\tau_{T}$ is interpreted as that caused by the microstructural interactions and is called the phase-lag of the temperature gradient. The other delay time $\tau_{q}$ is interpreted as the relaxation time due to the fast transient effects of thermal inertia and is called the phase-lag of the heat flux. The case when $\tau_{q}=\tau_{T}=0$, correspond to classical Fourier's law. If $\tau_{q}=\tau$ and $\tau_{T}=0$, Tzou refers to the model as single-phase-lag model. Roychoudhuri (2007) has studied one dimensional thermo-elastic wave propagation in an elastic half-space in the context of dual-phase-lag model. Recently, several researchers have attempted to solve their problems on the basis of the theory of dual-phase-lag model. Quintanilla $(2005,2006,2009)$ has solved several problems on the basis of this model. The exponential stability $(2002,2003)$ and condition of the delay parameters in the dual-phase-lag theory under this model have been studied by Quintanilla. Kumar, Prasad and Mukhopadhyay (2010) have studied the propagation of finite thermal wave in the context of dual-phase-lag model. he problem o finite thermal wave propagation in a half-space under variable thermal loading have been studied by Sur and Kanoria (2014). Recently, Karmakar et. al (2016) have studied the thermoelastic wave propagation in an infinite body having a spherical cavity under this theory. Also, a remarkable three-dimensional problem emplying this theory have been solved by Kalkal and Deswal (2014).

The objective of the present contribution is to consider a three-dimensional Kelvin-Voigt thermoviscoelastic medium in which, the bounding plane is subjected to prescribed tempertaure and boundary is free of tractions. The heat conduction equation has been formulated introducing dual-phase-lag heat model of heat conduction. Introducing the normal mode analysis, the governing equations have been expressed in cartesian coordiantes and are applied to a thermal shock problem in a viscoelastic body which fills the half-space. The numerical estimates of the thermophysical quantities have been computed for a copper-like material and have been depicted graphically to study the effect of viscosity on the physical quantities. The results corresponding to the cases when the effect of viscosity is unseen, agree with the results of the existing literature.

\section{Basic Equations}

The stress-strain-temperature relations are

$$
\sigma_{i j}=2 \mu^{*} e_{i j}+\left[\lambda^{*} \Delta-\gamma^{*} \theta\right] \delta_{i j}, \quad i, j=1,2,3
$$

where $\tau_{i j}$ is the stress tensor, $\theta_{0}$ is the reference temperature, $\theta$ is the temperature field, the cubical dilation, $\Delta=e_{i i}$ and $e_{i j}$ is the strain tensor given by

$$
e_{i j}=\frac{1}{2}\left(u_{i, j}+u_{j, i}\right) \text {. }
$$

Stress equation of motion in absence of body force is

$$
\sigma_{i j, j}=\rho \ddot{u}_{i}, \quad i, j=1,2,3
$$

The heat equation for the dynamic coupled generalized thermoviscoelasticity based on the dual-phaselag thermoelasticity model is given by

$$
K\left(1+\tau_{T} \frac{\partial}{\partial t}\right) \nabla^{2} \theta=\left(1+\tau_{q} \frac{\partial}{\partial t}+\frac{\tau_{q}^{2}}{2} \frac{\partial^{2}}{\partial t^{2}}\right)\left(\rho c_{v} \dot{\theta}+\gamma^{\mathrm{a}} \theta_{0} \dot{e}\right),
$$


where $u_{i}(i=1,2,3)$ are the displacement component and $\rho$ is the density, $c_{v}$ is the specific heat at constant strain and the parameters $\lambda^{\star}, \mu^{\star}$ and $\gamma^{\star}$ are defined as

$$
\lambda^{*}=\lambda_{e}\left(1+\alpha_{0} \frac{\partial}{\partial t}\right), \mu^{*}=\mu_{e}\left(1+\alpha_{0} \frac{\partial}{\partial t}\right), \gamma^{*}=\gamma_{e}\left(1+\alpha_{0} \frac{\partial}{\partial t}\right)
$$

where, $\alpha_{0}$ is the viscoelastic relaxation time, $\lambda_{e}, \mu_{e}$ being Lame's constant, $\gamma^{\star}=\left(3 \lambda^{\star}+2 \mu^{\star}\right) \alpha_{t} ; \alpha_{t}$

being the coefficient of linear thermal expansion. If $\tau_{T}=0, \tau_{q}=\tau_{0}$ and neglecting $\frac{\tau_{q}^{2}}{2}$, Eq. (4) reduces to the heat conduction equation corresponding to Lord-Shulman model; $\tau_{0}$ being the relaxation time for Lord-Shulman (LS) model.

\section{Formulation of the Problem}

We now consider an isotropic, homogeneous and thermoelastic medium with temperature dependent mechanical properties in three-dimensional space which fills the region $\Omega$, where $\omega$ is defined by $\Omega=\{(x, y, z): 0 \leq x \leq \infty,-\infty<y<\infty,-\infty<z<\infty\}$ subjected to a thermal shock on the bounding plane to the surface $x=0$. The body is initially at rest and the surface $x=0$ is assumed to be free of traction. Introducing the rectangular cartesian system $(x, y, z)$ having an origin on the surface $x=0$ with the $x$-axis vertical into the medium and the components of the displacement vector $\vec{u}$ as $(u, v, w)$. The constitutive relations are

$$
\begin{aligned}
& \sigma_{x x}=2 \mu_{e}\left(1+\alpha_{0} \frac{\partial}{\partial t}\right) \frac{\partial u}{\partial x}+\left[\lambda_{e}\left(1+\alpha_{0} \frac{\partial}{\partial t}\right) e-\gamma_{e}\left(1+\alpha_{0} \frac{\partial}{\partial t}\right) \theta\right] \\
& \sigma_{y y}=2 \mu_{e}\left(1+\alpha_{0} \frac{\partial}{\partial t}\right) \frac{\partial v}{\partial y}+\left[\lambda_{e}\left(1+\alpha_{0} \frac{\partial}{\partial t}\right) e-\gamma_{e}\left(1+\alpha_{0} \frac{\partial}{\partial t}\right) \theta\right] \\
& \sigma_{z z}=2 \mu_{e}\left(1+\alpha_{0} \frac{\partial}{\partial t}\right) \frac{\partial w}{\partial z}+\left[\lambda_{e}\left(1+\alpha_{0} \frac{\partial}{\partial t}\right) e-\gamma_{e}\left(1+\alpha_{0} \frac{\partial}{\partial t}\right) \theta\right] \\
& \sigma_{x y}=\mu_{e}\left(1+\alpha_{0} \frac{\partial}{\partial t}\right)\left(\frac{\partial u}{\partial y}+\frac{\partial v}{\partial x}\right) \\
& \sigma_{y z}=\mu_{e}\left(1+\alpha_{0} \frac{\partial}{\partial t}\right)\left(\frac{\partial v}{\partial z}+\frac{\partial w}{\partial y}\right) \\
& \sigma_{z x}=\mu_{e}\left(1+\alpha_{0} \frac{\partial}{\partial t}\right)\left(\frac{\partial u}{\partial z}+\frac{\partial w}{\partial x}\right) .
\end{aligned}
$$

The equations of motion in absence of body forces are

$$
\begin{aligned}
& \left(\lambda_{e}+2 \mu_{e}\right)\left(1+\alpha_{0} \frac{\partial}{\partial t}\right) \frac{\partial^{2} u}{\partial x^{2}}+\mu_{e}\left(1+\alpha_{0} \frac{\partial}{\partial t}\right)\left(\frac{\partial^{2} u}{\partial y^{2}}+\frac{\partial^{2} u}{\partial z^{2}}\right)+\left(\lambda_{e}+\mu_{e}\right)\left(1+\alpha_{0} \frac{\partial}{\partial t}\right)\left(\frac{\partial^{2} v}{\partial x \partial y}+\frac{\partial^{2} w}{\partial x \partial z}\right) \\
& -\gamma_{e}\left(1+\alpha_{0} \frac{\partial}{\partial t}\right) \frac{\partial \theta}{\partial x}=\rho \ddot{u}, \\
& \left(\lambda_{e}+2 \mu_{e}\right)\left(1+\alpha_{0} \frac{\partial}{\partial t}\right) \frac{\partial^{2} v}{\partial y^{2}}+\mu_{e}\left(1+\alpha_{0} \frac{\partial}{\partial t}\right)\left(\frac{\partial^{2} v}{\partial x^{2}}+\frac{\partial^{2} v}{\partial z^{2}}\right)+\left(\lambda_{e}+\mu_{e}\right)\left(1+\alpha_{0} \frac{\partial}{\partial t}\right)\left(\frac{\partial^{2} u}{\partial x \partial y}+\frac{\partial^{2} w}{\partial y \partial z}\right) \\
& -\gamma_{e}\left(1+\alpha_{0} \frac{\partial}{\partial t}\right) \frac{\partial \theta}{\partial y}=\rho \ddot{v},
\end{aligned}
$$




$$
\begin{aligned}
& \left(\lambda_{e}+2 \mu_{e}\right)\left(1+\alpha_{0} \frac{\partial}{\partial t}\right) \frac{\partial^{2} w}{\partial z^{2}}+\mu_{e}\left(1+\alpha_{0} \frac{\partial}{\partial t}\right)\left(\frac{\partial^{2} w}{\partial x^{2}}+\frac{\partial^{2} w}{\partial y^{2}}\right)+\left(\lambda_{e}+\mu_{e}\right)\left(1+\alpha_{0} \frac{\partial}{\partial t}\right)\left(\frac{\partial^{2} u}{\partial x \partial z}+\frac{\partial^{2} v}{\partial y \partial z}\right) \\
& -\gamma_{e}\left(1+\alpha_{0} \frac{\partial}{\partial t}\right) \frac{\partial \theta}{\partial z}=\rho \ddot{w} .
\end{aligned}
$$

The heat conduction equation for dual-phase-lag model is given by

$$
K\left(1+\tau_{T} \frac{\partial}{\partial t}\right)\left(\frac{\partial^{2} \theta}{\partial x^{2}}+\frac{\partial^{2} \theta}{\partial y^{2}}+\frac{\partial^{2} \theta}{\partial z^{2}}\right)=\left(1+\tau_{q} \frac{\partial}{\partial t}+\frac{\tau_{q}^{2}}{2} \frac{\partial^{2}}{\partial t^{2}}\right)\left[\rho c_{v} \dot{\theta}+\gamma_{e}\left(1+\alpha_{0} \frac{\partial}{\partial t}\right) \theta_{0} \frac{\partial}{\partial t}\left(\frac{\partial u}{\partial x}+\frac{\partial v}{\partial y}+\frac{\partial w}{\partial z}\right)\right]
$$

Eqs. (7-9) can be recast in the following form

$$
\begin{aligned}
& \rho \frac{\partial \ddot{u}}{\partial x}=\left(1+\alpha_{0} \frac{\partial}{\partial t}\right)\left[\mu_{e} \nabla^{2} \frac{\partial u}{\partial x}+\left(\lambda_{e}+\mu_{e}\right) \frac{\partial^{2} e}{\partial x^{2}}-\gamma_{e} \frac{\partial^{2} \theta}{\partial x^{2}}\right] \\
& \rho \frac{\partial \ddot{v}}{\partial y}=\left(1+\alpha_{0} \frac{\partial}{\partial t}\right)\left[\mu_{e} \nabla^{2} \frac{\partial v}{\partial y}+\left(\lambda_{e}+\mu_{e}\right) \frac{\partial^{2} e}{\partial y^{2}}-\gamma_{e} \frac{\partial^{2} \theta}{\partial y^{2}}\right], \\
& \rho \frac{\partial \ddot{w}}{\partial x}=\left(1+\alpha_{0} \frac{\partial}{\partial t}\right)\left[\mu_{e} \nabla^{2} \frac{\partial w}{\partial z}+\left(\lambda_{e}+\mu_{e}\right) \frac{\partial^{2} e}{\partial z^{2}}-\gamma_{e} \frac{\partial^{2} \theta}{\partial z^{2}}\right] .
\end{aligned}
$$

Introducing the following nondimensional variables

$$
\begin{aligned}
& x^{\prime}=c_{0} \eta x, \quad y^{\prime}=c_{0} \eta y, \quad z^{\prime}=c_{0} \eta z, \quad u^{\prime}=c_{0} \eta u, \quad v^{\prime}=c_{0} \eta v, \quad w^{\prime}=c_{0} \eta w, \quad t^{\prime}=c_{0}^{2} \eta t, \\
& \tau_{q^{\prime}}=c_{0}^{2} \eta \tau_{q}, \quad \tau_{T^{\prime}}=c_{0}^{2} \eta \tau_{T}, \quad \theta^{\prime}=\frac{\gamma_{e} \theta}{\rho c_{0}^{2}}, \quad \sigma_{i j}{ }^{\prime}=\frac{\sigma_{i j}}{\rho c_{0}^{2}}, \quad \alpha_{0^{\prime}}=c_{0}^{2} \eta \alpha_{0},
\end{aligned}
$$

where

$$
c_{0}^{2}=\frac{\lambda_{e}+2 \mu_{2}}{\rho} \quad \text { and } \quad \eta=\frac{\rho c_{v}}{K},
$$

and after removing primes, the above equations can be written in nondimensional form as follows

$$
\begin{aligned}
& \frac{\partial \ddot{u}}{\partial x}=\left(1+\alpha_{0} \frac{\partial}{\partial t}\right)\left[\delta \nabla^{2} \frac{\partial u}{\partial x}+(1-\delta) \frac{\partial^{2} e}{\partial x^{2}}-\frac{\partial^{2} \theta}{\partial x^{2}}\right] \\
& \frac{\partial \ddot{v}}{\partial y}=\left(1+\alpha_{0} \frac{\partial}{\partial t}\right)\left[\delta \nabla^{2} \frac{\partial v}{\partial y}+(1-\delta) \frac{\partial^{2} e}{\partial y^{2}}-\frac{\partial^{2} \theta}{\partial y^{2}}\right] \\
& \frac{\partial \ddot{w}}{\partial z}=\left(1+\alpha_{0} \frac{\partial}{\partial t}\right)\left[\delta \nabla^{2} \frac{\partial w}{\partial z}+(1-\delta) \frac{\partial^{2} e}{\partial z^{2}}-\frac{\partial^{2} \theta}{\partial z^{2}}\right] \\
& \left(1+\tau_{T} \frac{\partial}{\partial t}\right) \nabla^{2} \theta=\left(1+\tau_{q} \frac{\partial}{\partial t}+\frac{\tau_{q}^{2}}{2} \frac{\partial^{2}}{\partial t^{2}}\right)\left[\dot{\theta}+\delta_{0}\left(1+\alpha_{0} \frac{\partial}{\partial t}\right) \dot{e}\right]
\end{aligned}
$$

where

$$
\delta_{0}=\frac{\gamma_{e}^{2} \theta_{0}}{\rho c_{v}\left(\lambda_{e}+2 \mu_{e}\right)} \quad \text { and } \quad \delta=\frac{\mu_{e}}{\lambda_{e}+2 \mu_{e}} .
$$

In a similar manner, we can transform the constitutive relations in nondimensional forms. The dimensionless expressions for the constitutive are obtained by adding Eqs. (19-21) as follows, 


$$
\ddot{e}=\left(1+\alpha_{0} \frac{\partial}{\partial t}\right)\left[\nabla^{2} e-\nabla^{2} \theta\right]
$$

We shall consider the in variant stress $\sigma$ to be the mean value of the principal stresses as follows

$$
\sigma=\frac{\sigma_{x x}+\sigma_{y y}+\sigma_{z z}}{3} .
$$

Substituting the expressions of $\sigma_{x x}, \sigma_{y y}$ and $\sigma_{z z}$ into the above expressions, we obtain

$$
\sigma=\delta_{1}\left(1+\alpha_{0} \frac{\partial}{\partial t}\right) e-\left(1+\alpha_{0} \frac{\partial}{\partial t}\right) \theta
$$

where

$$
\delta_{1}=\frac{3-4 \delta}{3} .
$$

\section{Normal mode analysis}

In this method, the solutions of the physical variables can be decomposed in terms of normal modes in the following form

$$
\left[u, v, w, e, \theta, \sigma_{i j}\right](x, y, z, t)=\left[u^{\star}, v^{\star}, w^{\star}, e^{\star}, \theta^{\star}, \sigma_{i j}^{\star}\right](x) \exp [\omega t+i(m y+n z)]
$$

where $u^{\star}(x), v^{\star}(x), w^{\star}(x), e^{\star}(x), \theta^{\star}(x)$ and $\sigma_{i j}^{\star}(x)$ are the amplitudes of the functions $i=\sqrt{-1}$, $\omega$ is the angular frequency, $m$ and $n$ are the wave numbers in $y$ and $z$ directions respectively. Using the normal modes to Eqs. (22)-(24) and then eliminating $e^{\star}(x)$ from the resulting expressions, we obtain the system of ordinary differential equations

$$
\begin{aligned}
& \left(D^{2}-m^{2}-n^{2}\right) \theta^{*}(x)=\gamma_{1}\left[\left(1=\alpha_{0} \omega\right)\left(\delta_{0}+\delta_{1}\right) \theta^{*}(x)+\delta_{0} \sigma^{*}(x)\right], \\
& \left(D^{2}-m^{2}-n^{2}\right) \sigma^{\star}(x)=a_{1} \sigma^{\star}(x)+a_{2} \theta^{\star}(x) .
\end{aligned}
$$

where

$$
\begin{aligned}
& \gamma_{1}=\frac{\omega\left(1+\tau_{q} \omega+\frac{\tau_{q}^{2}}{2} \omega^{2}\right)}{\delta_{1}\left(1+\alpha_{0} \omega\right)\left(1+\omega \tau_{T}\right)}, \\
& a_{1}=\frac{\omega^{2}}{1+\alpha_{0} \omega}-\left(1-\delta_{1}\right)\left(1+\alpha_{0} \omega\right) \delta_{0} \gamma_{1} \text { and } a_{2}=\omega^{2}-\left(1-\delta_{1}\right)\left(1+\alpha_{0} \omega\right)^{2} \gamma_{1}\left(\delta_{0}+\delta_{1}\right) .
\end{aligned}
$$

Elimination of $\theta^{\star}(x)$ from Eq. (26) and Eq. (22) yield the following fourth-order differential equation

$$
\left(D^{4}-L D^{2}+M\right) \sigma^{\star}(x)=0,
$$

where

$$
L=\alpha_{1}+\alpha_{2}, \quad M=\alpha_{1} \alpha_{2}-a_{2} \gamma_{1} \delta_{0}, \quad \alpha_{1}=m^{2}+n^{2}+a_{1}, \quad \alpha_{2}=m^{2}+n^{2}+\left(\delta_{0}+\delta_{1}\right) \gamma_{1}\left(1+\alpha_{0} \omega\right) .
$$

Since the intent is that the solutions vanish at infinity so as to satisfy the regularity condition at infinity, we now cancel the exponential part that has positive power. Therefore, we have

$$
\sigma^{\star}(x)=\sum_{i=1}^{2} R_{i} e^{-\lambda_{i} x}
$$




$$
\theta^{\star}(x)=\sum_{i=1}^{2} R_{i^{\prime}} e^{-\lambda_{i} x}
$$

Using Eq. (29) and Eq. (30), we arrive from Eq. (24)

$$
e^{\star}(x)=\sum_{i=1}^{2}\left(d_{i}+\frac{1}{1+\alpha_{0} \omega}\right) R_{i} e^{-\lambda_{i} x}
$$

where

$$
R_{i^{\prime}}=d_{i} R_{i}, \quad \text { and } \quad d_{i}=\frac{\lambda_{i}^{2}-\alpha_{1}}{a_{2}} .
$$

\section{Application}

In order to determine the constants $R_{1}$ and $R_{2}$, we need to consider the following boundary conditions on the surface $x=0$ as follows

\section{(i) Thermal boundary condition}

The bounding plane $x=0$ is subjected to time-dependent thermal shock in the form

$$
\theta(0, y, z, t)=f(y, z, t)
$$

Therefore

$$
\theta(0, y, z, t)=f^{\star} e^{[\omega t+i(m y+n z)]} .
$$

\section{(ii) Mechanical boundary condition}

The bounding plane $x=0$ is free of traction. So, we have

$$
\sigma(x, y, z, t)=\sigma_{x x}(x, y, z, t)=\sigma_{y y}(x, y, z, t)=\sigma_{z z}(x, y, z, t)=0 \quad \text { on } \quad x=0 .
$$

Using Eq. (20), we have

$$
\sigma^{\star}(x)=\sigma_{x x}^{\star}(x)=\sigma_{y y}^{\star}(x)=\sigma_{z z}^{\star}(x)=0 \quad \text { on } \quad x=0 .
$$

Applying the boundary conditions to the above system of equations and solving the resulting system, we have

$$
R_{1}=\frac{f^{\star}}{d_{1}-d_{2}}, \quad R_{2}=-\frac{f^{\star}}{d_{1}-d_{2}} .
$$

Now, performing the normal mode analysis over Eq. (14) and using Eq. (30) and Eq. (31), we arrive the solution of the displacement as follows,

$$
u^{\star}(x)=R_{3} e^{-\lambda_{u} x}+\frac{l_{1} e^{-\lambda_{1} x}}{\lambda_{1}^{2}-\lambda_{u}^{2}}+\frac{l_{2} e^{-\lambda_{2} x}}{\lambda_{2}^{2}-\lambda_{u}^{2}}
$$

where

$$
\lambda_{u}^{2}=m^{2}+n^{2}+\frac{\omega^{2}}{\delta\left(1+\alpha_{0} \omega\right)}, \quad l_{i}=\frac{1}{\delta\left(1+\alpha_{0} \omega\right)}\left[\frac{1-\delta}{\delta_{1}}\left(d_{i}+\frac{1}{1+\alpha_{0} \omega}\right)-d_{i}\right] \lambda_{i} R_{i} .
$$


Also, $\lambda_{1}^{2} \neq \lambda_{2}^{2} \neq \lambda_{u}^{2}$ and $R_{3}$ is a constant to be determined. From Eq. (1) and Eq. (24), on using Eq. (25), we arrive at

$$
\sigma^{\star}(x)=2 \delta\left(1+\alpha_{0} \omega\right) D u^{\star}(x)+\frac{1-2 \delta}{\delta_{1}} \sigma^{\star}(x)+\left(1+\alpha_{0} \omega\right)\left(\frac{1-2 \delta-\delta_{1}}{\delta_{1}}\right) \theta^{\star}(x) .
$$

In view of the boundary conditions, the above equation transforms to

$D u^{\star}(x)=\left(\frac{\delta_{1}+2 \delta-1}{2 \delta \delta_{1}}\right) f^{\star} \quad$ on $\quad x=0$.

With the help of Eq. (35), we have

$$
R_{3}=\frac{1-2 \delta-\delta_{1}}{2 \delta \delta_{1} \lambda_{u}}-\frac{l_{1} \lambda_{1}}{\lambda_{u}\left(\lambda_{1}^{2}-\lambda_{u}^{2}\right)}-\frac{l_{2} \lambda_{2}}{\lambda_{u}\left(\lambda_{1}^{2}-\lambda_{u}^{2}\right)}
$$

Hence, the final solutions for the dimensionless stress $\sigma$, temperature $\theta$, strain $e$ and displacement $u$ can be deduced from Eqs. (29-31) and Eq. (35) as follows

$$
\begin{aligned}
& \sigma(x, y, z, t)=e^{\omega t} \cos (m y+n z)\left[R_{1} e^{-\lambda_{1} x}+R_{2} e^{-\lambda_{2} x}\right], \\
& \theta(x, y, z, t)=\frac{e^{\omega t} \cos (m y+n z)}{a_{2}}\left[\left(\lambda_{1}^{2}-\alpha_{1}\right) R_{1} e^{-\lambda_{1} x}+\left(\lambda_{2}^{2}-\alpha_{1}\right) R_{2} e^{-\lambda_{2} x}\right], \\
& e(x, y, z, t)=\frac{e^{\omega t} \cos (m y+n z)}{\delta_{1} a_{2}}\left[\left\{\left(\lambda_{1}^{2}-\alpha_{1}\right)+a_{2}\left(\alpha_{0} \omega+1\right)\right\} R_{1} e^{-\lambda_{1} x}+\left\{\left(\lambda_{2}^{2}-\alpha_{1}\right)+a_{2}\left(\alpha_{0} \omega+1\right)\right\} R_{2} e^{-\lambda_{2} x}\right], \\
& u(x, y, z, t)=e^{\omega t} \cos (m y+n z)\left[R_{3} e^{-\lambda_{u} x}+\frac{l_{1}}{\lambda_{1}^{2}-\lambda_{u}^{2}} e^{-\lambda_{1} x}+\frac{l_{2}}{\lambda_{2}^{2}-\lambda_{u}^{2}} e^{-\lambda_{2} x}\right] .
\end{aligned}
$$

\section{Numerical results and discussions}

With an aim to illustrate the results obtained in the preceding section, we now present the analytical results numerically. In the numerical computation, we have considered a copper-like material. Since $\omega$ is the complex time constant, we have $\omega=\omega_{0}+i \zeta$, then $e^{\omega t}=e^{\omega_{0} t}(\cos \zeta t+i \sin \zeta t)$. The values of the material constants are taken to be (Kalkal and Deswal, 2014)

$$
\begin{gathered}
\rho=8954 \mathrm{~kg} \cdot \mathrm{m}^{-3}, \quad K=386 \mathrm{~W} \cdot \mathrm{m}^{-1} \cdot \mathrm{K}^{-1}, \quad v=0.33, \quad T_{0}=293 \mathrm{~K}, \quad \alpha_{t}=1.78 \times 10^{-5}, \\
c_{v}=383.1 \mathrm{~J} \cdot \mathrm{kg}^{-1} \cdot \mathrm{K}^{-1}, \quad \tau_{T}=0.15 \mathrm{~s}, \quad \tau_{0}=\tau_{q}=0.2 \mathrm{~s}, \quad \alpha=0.001 \mathrm{~K}^{-1} \\
\omega_{0}=1.0, \quad \zeta=0.2, \quad \alpha_{0}=0.1 .
\end{gathered}
$$

Further, the values of the other non-dimensional parameters arising in the present analysis are taken to be

$$
f^{\star}=10, \quad m=1.2, \quad n=1.3 .
$$

In order to study the effect of viscosity on the thermophysical quantities for LS and DP lag models, Figs. (1-4) have been plotted. In these figs., the continuous lines represent the graphs corresponding to nonviscous (NVIS) model and the dotted lines corresponds to that for a viscous (VIS) medium. 


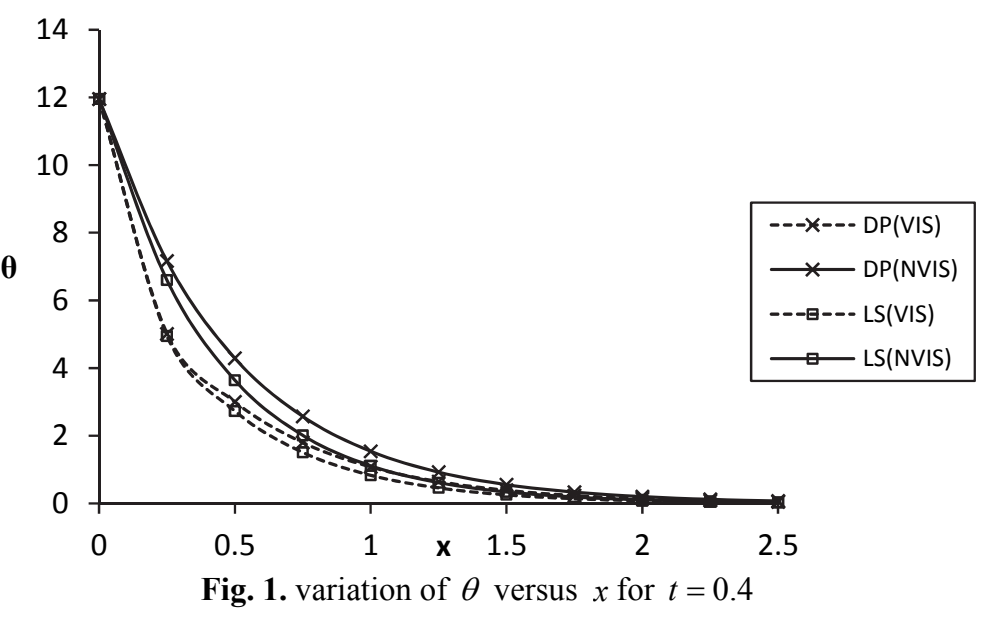

Fig. 1 depicts the variation of the temperature $\theta$ against the distance $x$ when $y=z=1.0$ and the time $t=0.4$. As seen from the figure, $\theta$ attains its maximum magnitude on the plane $x=0$, i.e., near the plane of application of the thermal loading, which complies with the real situation, and it decays for larger value of $x$. Due to the present of viscosity term, the magnitude of $\theta$ is lesser than that corresponding to a nonviscous medium in $0<x<2.5$. Further, the rate of decay of $\theta$ is faster in LS model than that of DP lag model. This phenomenon is observed for both viscous and nonviscous medium.

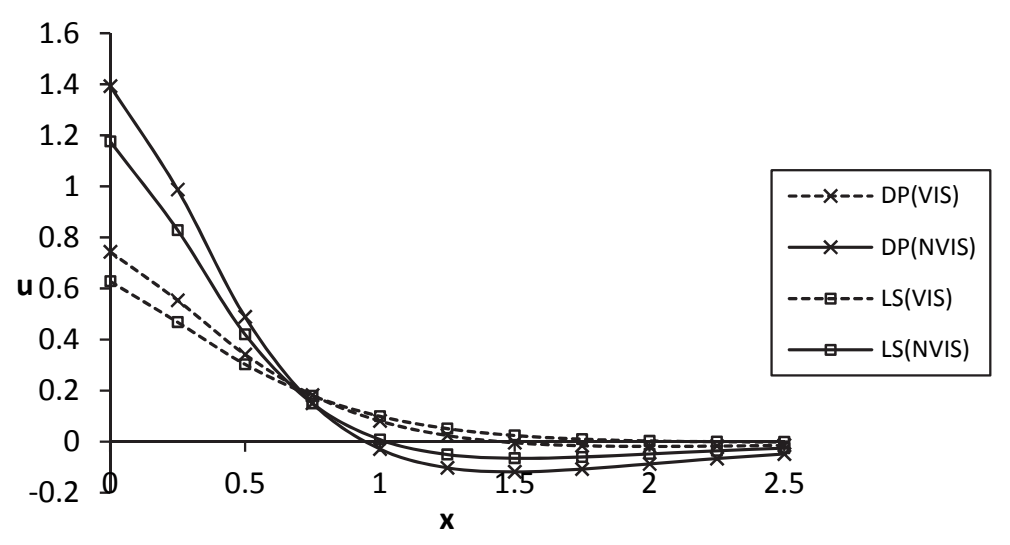

Fig. 2. Variation of $u$ versus $x$ for $t=0.4$

Fig. 2 depicts the variation of the displacement component $u$ against the distance $x$ when $y=z=1.0$ and $t=0.4$ for both viscous and nonviscous medium. It is observed that the displacement field starts with a maximum value in all the figures on the plane $x=0$ and it almost diminishes for a large value of $x$. The decay of the displacement component $u$ is faster for nonviscous medium than that of the viscous medium. Also, for both viscous and nonviscous medium, the magnitude of $u$ is larger for DP model than that of LS model and finally it diminishes to zero at far from the point of application of the thermal loading.

Fig. 3 depicts the variation of thermal stress $\sigma$ against the distance $x$ for the same set of parameters. It is observed that on the plane $x=0, \sigma$ vanishes satisfying the mechanical boundary condition. Further, the stress field increases sharply in $0<x<0.5$ to attain its maximum value at $x=0.5$ and then it diminishes approximately to zero value for a large value of $x$. The figure also reveal the fact that due to the presence of the viscosity term, the magnitude of $\sigma$ decays than that for nonviscous medium. Further, for both viscous and nonviscous medium, the magnitude of stress eave is larger for DP model than that of LS model. 


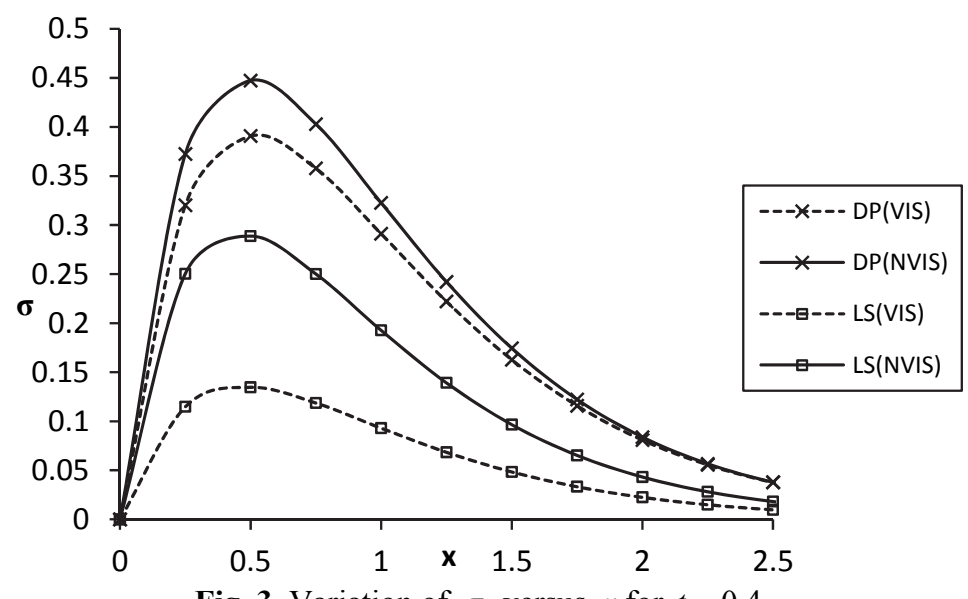

Fig. 3. Variation of $\sigma$ versus $x$ for $t=0.4$

Fig. 4 has been plotted to study the variation of the elongation $e$ against the distance $x$. For both viscous and nonviscous medium, the stress field follows a similar trend only having differences in magnitude. It is observed that the strain field has maximum magnitude near the vicinity of the application of thermal loading which is physically plausible and then it diminishes to zero as $x$ diverges from the point of the source application. The strain also shows significant sensitivity on the effect of viscosity. For both the models, the magnitude of the elongation diminishes due to the presence of the viscosity term.

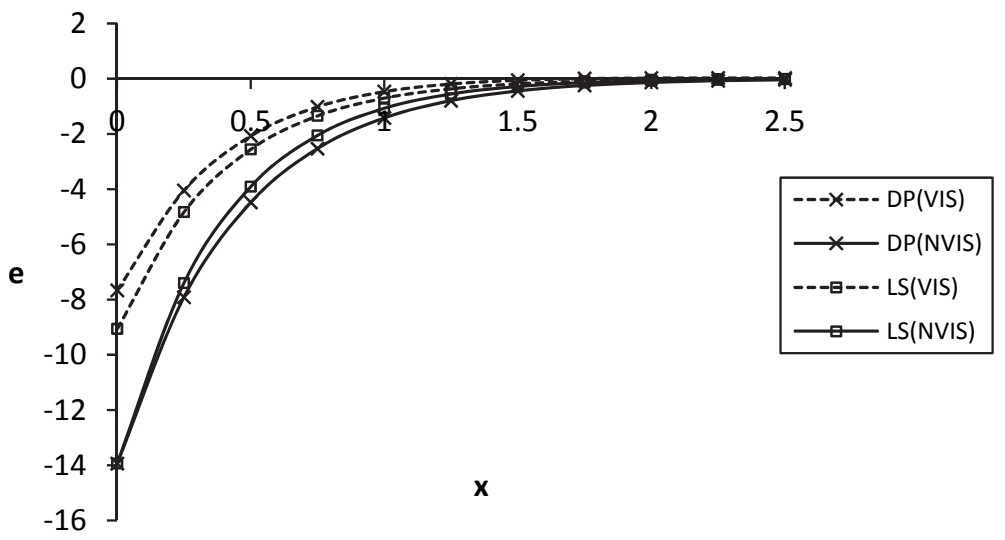

Fig. 4. Variation of $e$ versus $x$ for $t=0.4$

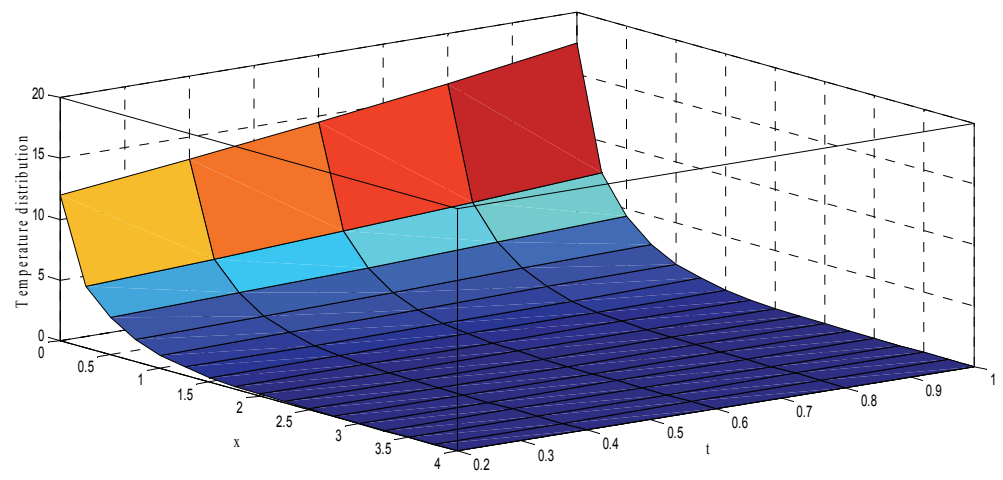

Fig. 5. Profile of $\theta$ versus $x$ and $t$ for viscous medium 
Figs. 5-8 have been plotted to study the profile of the thermophysical quantities for a viscous medium when $y=z=1.0$ and for different length $x$ and time $t$ respectively.

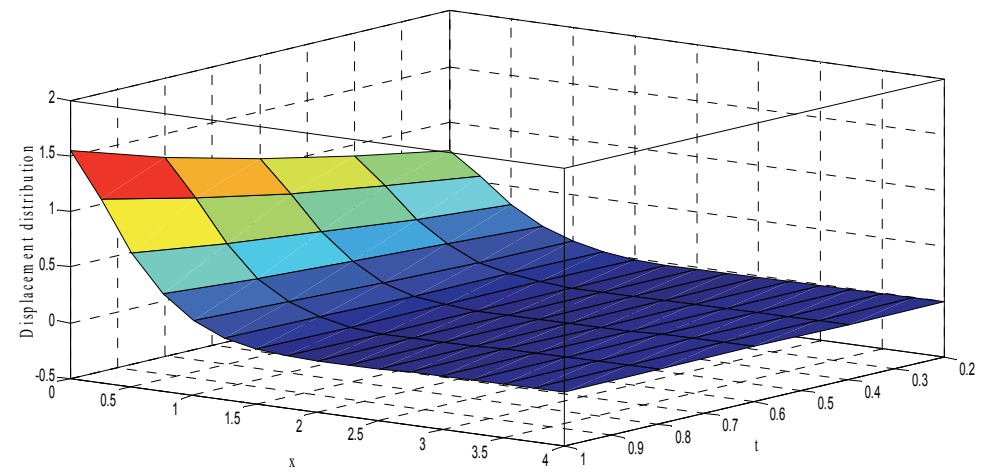

Fig. 6. Profile of $u$ versus $x$ and $t$ for viscous medium

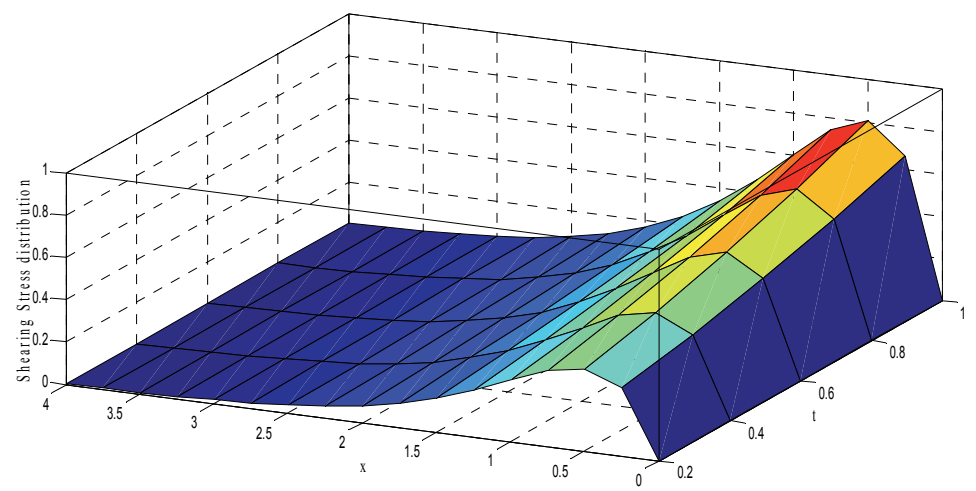

Fig. 7. Profile of $\sigma$ versus $x$ and $t$ for viscous medium

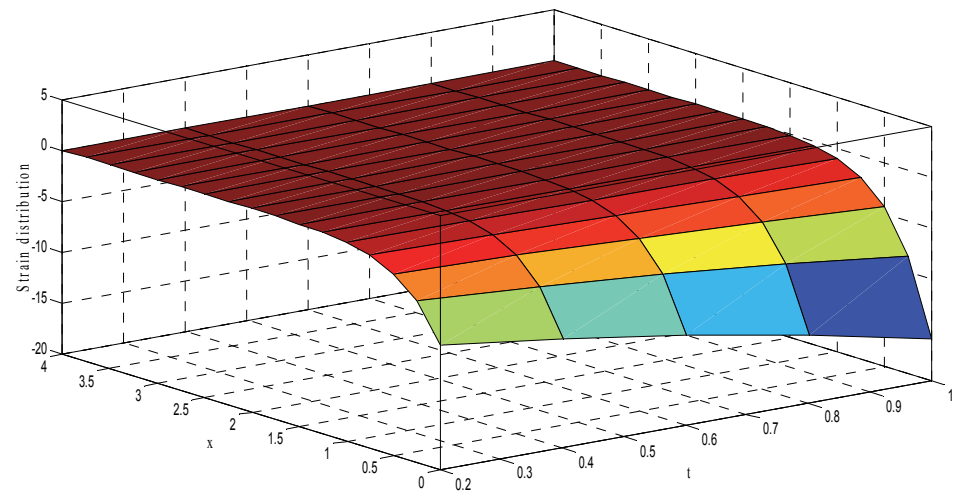

Fig. 8. Profile of $e$ versus $x$ and $t$ for viscous medium

As seen from these figs., with the increase of the time $t$, the magnitude of the thermophysical quantities with also increase which is quite plausible. 


\section{Conclusions}

In this present analysis, a mathematical treatment has been presented to explore the effect of viscosity on wave propagation in a three-dimensional generalized Kelvin-Voigt thermoelastc model under dual-phase lags. The problem has been solved theoretically and exemplified through specific models (LS and DP models). All the figures plotted are self-explanatory in exhibiting the different peculiarities which occur in the propagation of waves, yet the following remarks may be added.

1. The presence of the thermo-viscoelastic relaxation parameters will decrease the magnitude of the thermophyscial quantities and the decay of the physical quantities becomes slower due to the presence of the viscosity term. So, the parameter of viscosity have a significant effect on all the thermophysical quantities.

2. From the distribution of the temperature, we see that the thermal wave is propagating with a finite speed which validates the hyperbolic thermoelasticity theory.

3. Here all the results for viscosity parameter $\alpha_{0}=0$ and for Dual-phase lag model, agree with the existing literature.

\section{Acknowledgements}

We are grateful to Prof. S. C. Bose of the Department of Applied Mathematics, University of Calcutta for his valuable suggestions and guidance in preparation of the paper.

\section{References}

Alfery, T., \& Gurnee, E. F. (1956). In: Eirich, F.R. (Ed.), In Rheology: Theory and Applications, vol. 1. Academic Press, New York.

Bandyopadhyay, N., \& Roychoudhuri, S. K. (2005). Thermoelastic wave propagation without energy dissipation in an elastic half space. Bulletin of Calcutta Mathematical Society, 97(6), 489-502.

Banik, S., Mallik, S. H., \& Kanoria, M. (2007). Thermoelastic interaction with energy dissipation in an infinite solid with distributed periodically varying heat sources. International Journal of Pure and Applied Mathematics, 34(2), 231-245.

Banik, S., Mallik, S. H., \& Kanoria, M. (2009). A unified generalized thermoelasticity formulation: application to an infinite body with a cylindrical cavity and variable material properties. International Journal of Applied Mechanics and Engineering, 14(1), 113-126.

Bland, D. R. (1960). The Theory of Linear Viscoelasticity. Pergamon Press, Oxford.

Biot, M. A. (1954). Theory of stress-strain relations in an isotropic viscoelasticity and relaxation phenomena. Journal of Applied Physics, 25(11), 1385-1391.

Biot, M. A. (1955). Variational principal in irreversible thermodynamics with application to viscoelasticity. Physics Review, 97(6), 1463-1469.

Biot, M. A. (1956). Thermoelasticity and irreversible thermodynamics. Journal of Applied Physics, 27(3), 240-253.

Chandrasekharaiah, D. S. (1986). Thermoelasticty with second sound; A review. Applied Mechanics Review, 39(3), 355-375.

Chandrasekharaiah, D. S. (1996a). A uniqeness theorem in the theory of thermoelasticity without energy dissipation. Journal of Thermal Stresses, 19(3), 267-272.

Chandrasekharaiah, D. S. (1996b). A note on the uniqueness of solution in the linear theory of thermoelasticity without energy dissipation. Journal of Elasticity, 43(3), 279-283.

Chandrasekharaiah, D. S. (1998). Hyperbolic thermoelasticity: A review of recent literature. Applied Mechanics Review, 51(12), 705-729.

Ferry, J. D. (1970). Viscoelastic Properties of Polymers. J. Wiley and Sons, New York. 
Freudenthal, A. M. (1954). Effect of rheological behaviour on thermal stress. Journal of Applied Physics, 25(9), 1-10.

Green, A. E., \& Lindsay, K. A. (1972). Thermoelasicity. Journal of Elasticity, 2, 1-7.

Green, A. E., \& Laws, N. (1972). On the entropy production inequality. Archive for Rational Mechanics and Analysis, 45(1), 47-53.

Green, A. E., \& Naghdi, P. M. (1991). A re-examination of the basic postulates of thermomechanics. Proceedings of Royal Society, Series A 432, 171-194.

Green, A. E., Naghdi, P. M. (1993). Thermoelasticity without energy dissipation. Journal of Elasticity, 31(3), 189-208.

Gross, B. (1953). Mathematical structure of the theories of viscoelasticity. Hermann: Paris.

Gurtin, M. E., \& Sternberg, E. (1962). On the linear theory of viscoelasticity. Archive for Rational Mechanics and Analysis, 11(1), 291-356.

Hetnarski, R. B., Ignaczak, J. (1996). Soliton-like waves in a low-temperature nonlinear thermoelastic solid. International Journal of Engineering Science, 34(15), 1767-1787.

Hetnarski, R. B., \& Ignaczak, J. (1999). Generalized thermoelasticity. Journal of Thermal Stresses, 22(4-5), 451-476.

Ignaczak, J. (1989). Generalized thermoelasticity and its applications. Thermal Stresses III, chap. 4. In: Hetnarski, R.B. (Ed.), Mechanical and Mathematical Methods. North Holland.

Iiioushin, A. A., \& Pobedria, B. E. (1970). Mathematical Theory of Thermal Viscoelasticity. Nauka, Moscow.

Kalkal, K. K., \& Deswal, S. (2014). Effects of phase-lags on three-dimensional wave propagation with temperature dependent properties. International Journal of Thermophysics, 35, 952-969.

Kar, A., \& Kanoria, M. (2009b). Generalized thermoe-visco-elastic problem of a spherical shell with three-phase-lag effect. Applied Mathematical Modelling, 33, 3287-3298.

Karmakar, R., Sur, A., \& Kanoria, M. (2016). Thermoelastic interaction in an infinite body under dual phase lags. Journal of Applied Mechanics and Technical Physics, In Press.

Kosinski, W., \& Cimmelli, V. A. (1997). Gradient generalization to inertial state variables and a theory of super fluidity. Journal of Theoretical and Applied Mechanics, 35, 763-779.

Kosinski, W. (1989). Elastic Waves in the Presence of a New Temperature Scale, in Elastic Wave Propagation. Elsevier, New York, 629-634.

Lakes, R. S. (1998). Viscoelastic Solids. CRC Press, New York.

Lord, H. W., \& Shulman, Y. H. (1967). A generalized dynamical theory of thermoelasticity. Journal of Mechanics and Physics of Solids, 15(5), 299-309.

Mallik, S. H., \& Kanoria, M. (2007). Generalized thermoelastic functionally graded solid with a periodically varying heat source. International Journal of Solids and Structures, 44(22-23), 76337645.

Mallik, S. H., \& Kanoria, M. (2008). A two dimensional Problem for a transversely isotropic generalized thermoelastic thick plate with spatially varying heat source. European Journal of Mechanics A/Solids, 27(4), 607-621.

Mallik, S. H., Kanoria, M. (2009). A unified generalized thermoelasticity formulation: application to penny shaped crack analysis. Journal of Thermal Stresses, 32(9), 1-23.

Muller, I. M. (1971). The Coldness, a universal function in thermoelastic bodies. Archive for Rational Mechanics and Analysis, 41(5), 319-332.

Prasad, R., Kumar, R., \& Mukhopadhyay, S. (2010). Propagation of harmonic plane waves under thermoelasticity with dual-phase-lags. International Journal of Engineering Science, 48(12), 20282043.

Quintanilla, R., \& Horgan, C. O. (2005). Spatial behaviour of solutions of the dual-phase-lag heat equation. Mathematical Methods in Applied Sciences, 28(1), 43-57.

Quintanilla, R, Racke, R. (2006) A note on stability in dual-phase-lag heat conduction. International Journal of Heat and Mass Transfer, 49(7), 1209-1213.

Quintanilla, R. (2009). A well-posed problem for the three-dual-phase-lag heat conduction. Journal of Thermal Stresses, 32(12), 1270-1278. 
Quintanilla, R. (2002). Exponential stability in the dual-phase-lag heat conduction theory. Journal of Non-Equilibrium Thermodynamics, 27(3), 217-227.

Quintanilla, R. (2003). A condition on the delay parameters in the one-dimensional dual-phase-lag thermoelastic theory. Journal of Thermal Stresses, 26(7), 713-721.

Roychoudhuri, S. K., \& Bandyopadhyay, N. (2005). Thermoelastic wave propagation in rotating elastic medium without energy dissipation. International Journal of Mathematics and Mathematical Sciences, 1, 99-107.

Roychoudhuri, S. K., \& Dutta, P. S. (2005). Thermoelastic interaction without energy dissipation in an infinite solid with distributed periodically varying heat sources. International Journal of Solids and Structures, 42(14), 4192-4203.

Roychoudhury, S. K. (2007). One-dimensional thermoelastic waves in elastic half-space with dualphase-lag effect. Journal of Mechanics of Materials and Structures, 2(3), 489-502.

Suhubi, E. S. (1975). Thermoelastic solids (Chapter 2). In: Eringen, A.C. (Ed.), Continuum Physics, Part 2, vol. 2. Academic Press, New York.

Sur A., \& Kanoria, M. (2014a). Thermoelastic interaction in a viscoelastic functionally graded halfspace under three-phase-lag model. European Journal of Computational Mechanics, 23(5-6), 179198.

Sur A., \& Kanoria, M. (2014b). Fractional heat conduction with finite wave speed in a thermo-viscoelastic spherical shell. Latin American Journal of Solids and Structures, 11(7), 1132-1162.

Sur, A., \& Kanoria, M. (2014). Finite thermal wave propagation in a half-space due to variable thermal loading. Applications and Applied Mathematics, 9(1), 94-120.

Taheri, H., Fariborz, S. J., \& Eslami, M. R. (2005). Thermoelastic analysis of an annulus using the Green-Naghdi model. Journal of Thermal Stresses, 28(9), 911-927.

Tanner, R. I. (1988). Engineering Rheology. Oxford University Press.

Tzou, D. Y. (1995). A unified field approach for heat conduction from macro to micro scales. ASME Journal of Heat Transfer, 117(1), 8-16. 